\title{
Cation distribution in natural ferrites
}

\author{
H.A. Maia, F.F.T. De Araújo, M.A.B. De Araújo, J. Danon ${ }^{1, \dagger}$ and R.B. Frankel ${ }^{2}$ \\ Departamento de Física, Universidade Federal do Ceará, Caixa Postal 6030. \\ 60.450 Fortaleza, CE-Brazil \\ ${ }^{1}$ Centro Brasileiro de Pesquisas Físicas, 22.290 Rio de Janeiro, Brazil \\ ${ }^{2}$ Physics Department, California Polytechnic State University, San Luis Obispo, \\ CA 93407, USA
}

Mössbauer spectra of jacobsite have been recorded at temperatures of 4.2, 125, 180, 240 and $300 \mathrm{~K}$ and with an external magnetic field of $60 \mathrm{kOe}$ applied parallel to the incident radiation at $4.2 \mathrm{~K}$. The results show the presence of two different magnetic hyperfine interactions associated with the iron nuclei. Spectra of samples in external magnetic fields indicate the same sign for the magnetic hyperfine interactions, implying that both iron ions occupy only the octahedral sublattice. These results are compared with data from synthetic stoichiometry manganese ferrite, and a model of cation distribution, assuming the existence of vacancies, is proposed. According to this model, the mineral jacobsite is rich in manganese compared with stoichiometric $\mathrm{MnFe}_{2} \mathrm{O}_{4}$ ferrite.

\section{Introduction}

In the past years, attention has been given to materials with spinel structures such as ferrites which have the general formula $(\mathrm{A}, \mathrm{Fe})_{3} \mathrm{O}_{4}$, where $\mathrm{A}$ is any bivalent cation of the first transition element group. Among these materials are the manganese ferrites $(A=M n)$, which have been extensively investigated due to their utilization in electronics and also because their magnetic and electronic properties are strongly affected by the cationic composition [1-6]. However, investigations have been restricted only to synthetic manganese ferrites, and there are no publications regarding natural manganese ferrite. In this work, we report on the study of the natural manganese ferrite known as jacobsite, which occurs in the northeast of Brazil. Although data from other techniques, such as magnetization and X-ray fluorescence have also been used, this study is based mainly on the Mössbauer spectroscopy results.

\footnotetext{
${ }^{\dagger}$ This paper is dedicated to the memory of Professor Jacques Danon, who was the pioneer of Mössbauer spectroscopy in Brazil.
} 


\section{Experimental}

The samples were collected, ground into very fine powder and, making use of a permanent bar magnet, the powder was separated into magnetic and nonmagnetic components. This separation process was done in various steps in order to ensure the complete separation of the components. The weight percentages of the jacobsite under study were about $32 \%$ magnetic and $68 \%$ non-magnetic. In this work, we only dealt with the magnetic component.

According to $\mathrm{X}$-ray fluorescence data, the atomic ratio $\mathrm{Mn} / \mathrm{Fe}$ was of the order of 1.25 for the magnetic component. X-ray powder diffraction analysis confirmed the presence of a reasonable amount of the manganese ferrite $\left(\mathrm{MnFe}_{2} \mathrm{O}_{4}\right)$ and also small quantities of the silicate $\mathrm{Mn}_{2} \mathrm{SiO}_{4}$ and manganese oxide $\mathrm{MnO}_{2}$. The magnetic moment was measured at $4.2 \mathrm{~K}$ with a vibrating sample magnetometer. The Mössbauer measurements were performed using a conventional constant acceleration spectrometer operating in the triangular wave mode in transmission geometry. The Mössbauer parameters were determined by computer fitting using a least-squares routine.

\section{Results and discussion}

A series of Mössbauer spectra of jacobsite recorded at 300,240,180, 125 and $4.2 \mathrm{~K}$ in zero applied magnetic field is shown in fig. 1. All of these spectra were fitted with two sextets. For comparison, a room temperature Mössbauer spectrum of a synthetic manganese ferrite taken from ref. [4] is presented in fig. 2. Table 1 lists the Mössbauer parameters obtained from the fits of the spectra of fig. 1. The symbols (1) and (2) represent the high and low intensity sextets, respectively.

Using neutron diffraction results, Hastings and Carliss [1] proposed the cation distribution $\left(\mathrm{Mn}_{0.8}^{2+} \mathrm{Fe}_{0.2}^{3+}\right)\left[\mathrm{Mn}_{0.2}^{2+} \mathrm{Fe}_{1.8}^{3+}\right] \mathrm{O}_{4}^{2-}$ for a stoichiometric synthetic manganese ferrite. According to this distrbution, the sextets of the Mössbauer spectrum of the synthetic ferrite can be ascribed to $10 \%$ of $\mathrm{Fe}^{3+}$ in the tetrahedral site (round brackets) and to $90 \%$ of $\mathrm{Fe}^{3+}$ in the octahedral site (square brackets). However, there are visible differences between the spectra of jacobsite and the spectrum of synthetic manganese ferrite. The spectrum of jacobsite recorded in zero applied magnetic field shows that the low intensity sextet has the smaller magnetic hyperfine field, whereas the spectrum of synthetic ferrite shows that the low intensity sextet has the larger magnetic hyperfine field. Figure 3 displays the spectrum of jacobsite recorded at $4.2 \mathrm{~K}$ under an external field of $60 \mathrm{kOe}$ applied parallel to the $\gamma$ radiation. Again for comparison, a $7 \mathrm{~K}$ spectrum of synthetic manganese ferrite, recorded in an external field of $55 \mathrm{kOe}$ also applied parallel to the $\gamma$-radiation, is shown in fig. 4 . It is clearly observable in fig. 4 that the applied field adds to the hyperfine field associated with the low intensity sextet, and subtracts from the hyperfine field associated with the high intensity sextet. This means that the two sextets correspond to iron ions with antiparallel magnetic hyperfine fields. Moreover, as the neutron diffraction results $[7,8]$ indicate that the net magnetic moment of the 


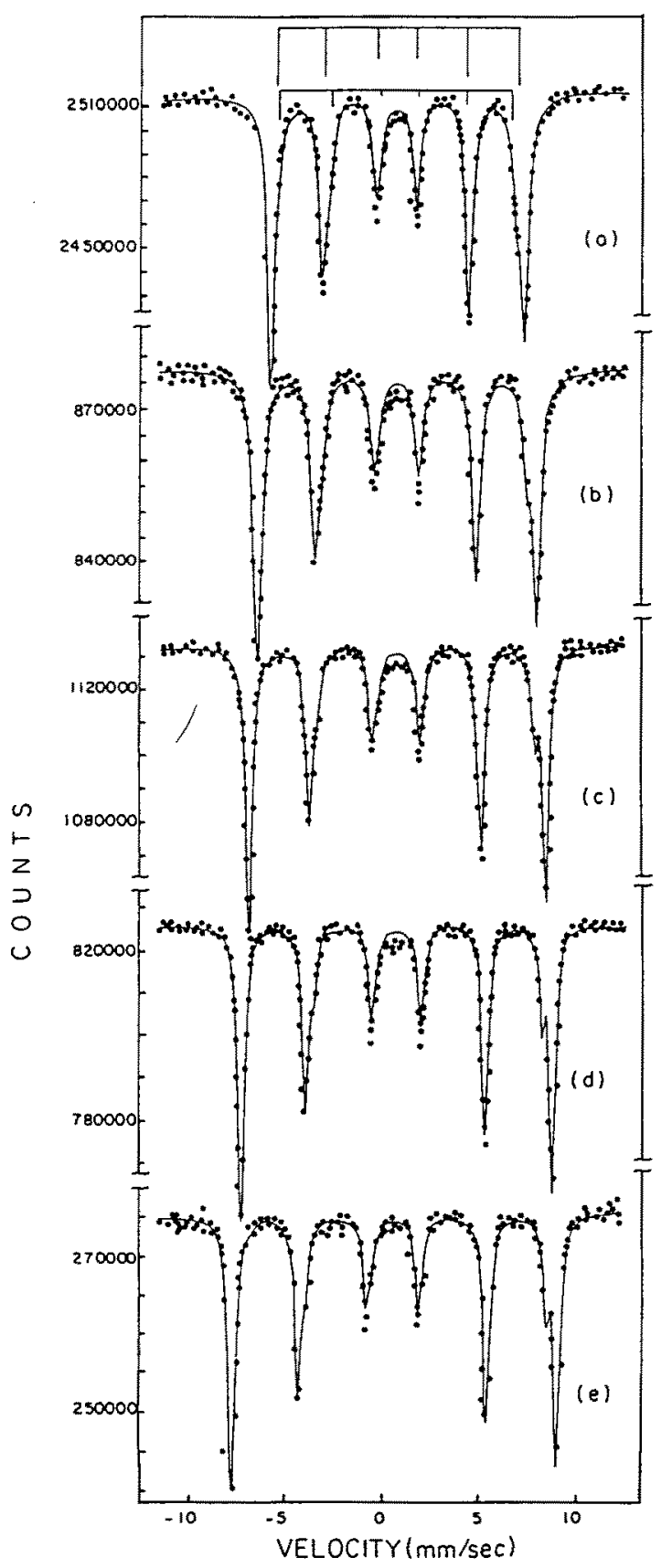

Fig. 1. Mössbauer spectra of jacobsite recorded at (a) room temperature, (b) $240 \mathrm{~K}$. (c) $180 \mathrm{~K}$, (d) $125 \mathrm{~K}$, (e) $4.2 \mathrm{~K}$. 


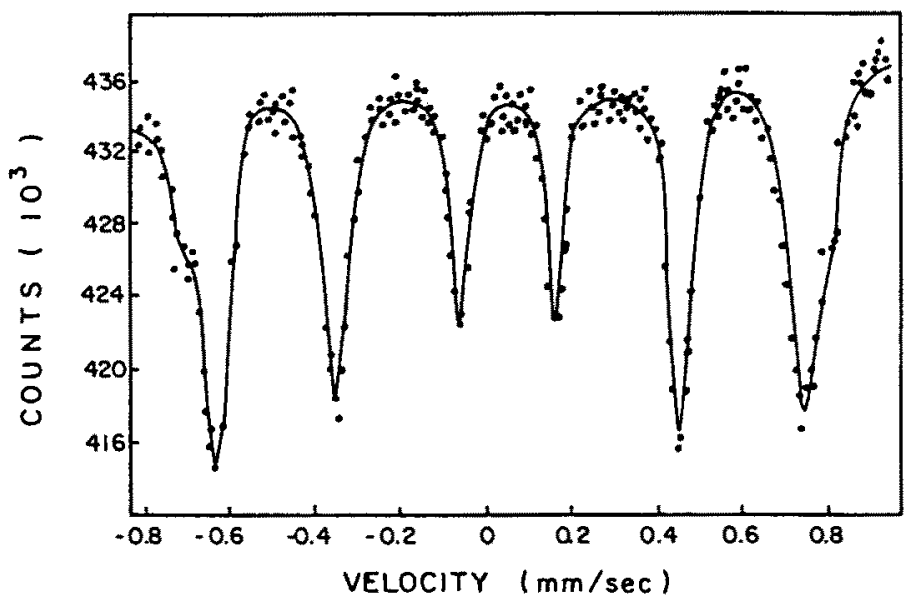

Fig. 2. Room temperature Mössbauer spectrum of synthetic manganese ferrite.

Table 1

Isomer shift $(\delta)$ relative to metallic iron, quadrupole splitting $(\Delta E q)$ and effective magnetic hyperfine field $(H)$ values for jacobsite at various temperatures. (The estimated error for these parameters is $\pm 3 \%$.)

\begin{tabular}{cccccccc}
\hline$T(\mathrm{~K})$ & $\begin{array}{c}\delta(1) \\
(\mathrm{mm} / \mathrm{s})\end{array}$ & $\begin{array}{c}\delta(2) \\
(\mathrm{mm} / \mathrm{s})\end{array}$ & $\begin{array}{c}\Delta E q(1) \\
(\mathrm{mm} / \mathrm{s})\end{array}$ & $\begin{array}{c}\Delta E q(2) \\
(\mathrm{mm} / \mathrm{s})\end{array}$ & $\begin{array}{c}H(1) \\
(\mathrm{kOe})\end{array}$ & $\begin{array}{c}H(2) \\
(\mathrm{kOe})\end{array}$ & $A_{1} / A_{2}$ \\
\hline 300 & 0.22 & 0.21 & 0.10 & 0.17 & 419 & 400 & 2.7 \\
240 & 0.27 & 0.21 & 0.15 & 0.44 & 455 & 444 & 3.1 \\
180 & 0.30 & 0.25 & 0.15 & 0.41 & 486 & 473 & 3.0 \\
125 & 0.32 & 0.27 & 0.15 & 0.36 & 509 & 496 & 3.0 \\
4.2 & 0.34 & 0.29 & 0.14 & 0.37 & 524 & 512 & 2.7 \\
\hline
\end{tabular}

octahedral sublattice is parallel to the applied magnetic field, it is easy to conclude that the high intensity sextet is associated with iron ions in octahedral sites and the low intensity sextet with ions in tetrahedral sites. The spectrum of jacobsite displayed in fig. 3 shows that the applied magnetic field subtracts from both magnetic hyperfine fields. Therefore, the spectrum of jacobsite must be interpreted in terms of iron ions situated only in octahedral sites with parallel magnetic moments. As a consequence, the tetrahedral site in jacobsite must be fully occupied by manganese ions. This is typical of manganese-rich ferrites [3]. It is also noticeable from the spectra shown in figs. 3 and 4 that the middle $(\Delta m=0)$ lines have vanished, implying that for both ferrites the spins order in a collinear arrangement. 


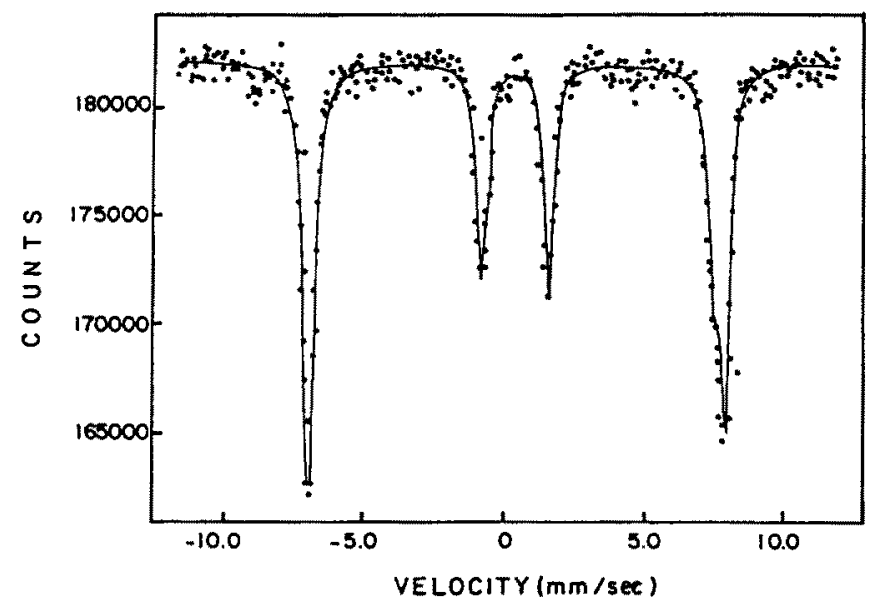

Fig. 3. Mössbauer spectrum of jacobsite recorded at $4.2 \mathrm{~K}$ in an external magnetic field of $60 \mathrm{kOe}$ applied parallel to the $\gamma$-radiation.

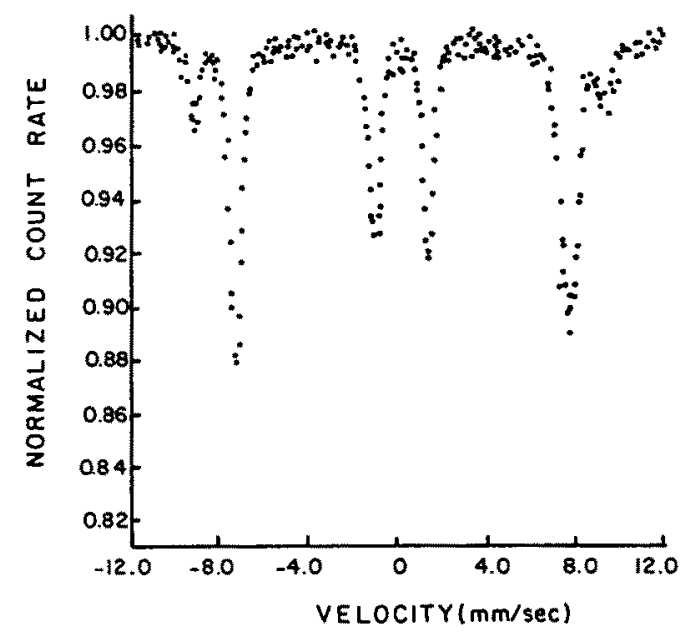

Fig. 4. Mössbauer spectrum of synthetic manganese ferrite recorded at $7 \mathrm{~K}$ in an external magnetic field of $55 \mathrm{kOe}$ applied parallel to the $\gamma$-radiation.

For manganese-rich ferrites, Eschenfelder [3] has proposed the cation distribution $\left(\mathrm{Mn}^{2+}\right)\left[\mathrm{Mn}_{x-1}^{4+} \mathrm{Fe}_{x-1}^{2+} \mathrm{Fe}_{4-2 x}^{3+}\right] \mathrm{O}_{4}^{2-}$, with $1<x<2$. In principle, this distribution would explain the existence of two sextets in the jacobsite spectrum, associated with iron in octahedral sites. However, the Mössbauer parameters of jacobsite are typical of only $\mathrm{Fe}^{3+}$. Further, the low temperature spectra show no evidence of any component in addition to the $\mathrm{Fe}^{3+}$, and the area ratio $A_{1} / A_{2}$ remains nearly constant with 
decreasing temperature, thus excluding the possibility of electron hopping processes. Therefore, the cationic composition proposed by Eschenfelder [3] is not suitable for jacobsite. Another cation distribution which has been frequently used for manganeserich ferrites [2,9-11] is $\left(\mathrm{Mn}_{1-\varepsilon}^{2+} \mathrm{Fe}_{\varepsilon}^{3+}\right)\left[\mathrm{Mn}_{x-1}^{3+} \mathrm{Mn}_{\varepsilon}^{2+} \mathrm{Fe}_{3-x-\varepsilon}^{3+}\right] \mathrm{O}_{4}^{2-}$, where $x>1$. For $\varepsilon=0$, this distribution fits our experimental results, provided we find an explanation for the existence of two sextets associated only with $\mathrm{Fe}^{3+}$ in the octahedral site.

In looking at the Mössbauer parameters listed in table 1, one can see that there is a temperature dependence of isomer shift $(\delta)$ and quadrupole splitting $(\Delta E q)$. The changes in the isomer shifts are easily explained by the second-order Doppler effect $[12,13]$. For the quadrupole splitting, there is an especially large variation in $\Delta E q(2)$, by a factor of 3 , between 300 and $180 \mathrm{~K}$. Since all the $\mathrm{Fe}^{3+}$ ions occupy octahedral sites, we must now explain not just the existence of two sextets but also the temperature dependence of $\Delta E q(2)$. One possible explanation is based on the existence of $\mathrm{O}^{2-}$ vacancies at some of the octahedral sites. At high temperature $(300 \mathrm{~K})$, the vacancies are thermally excited and move rapidly, resulting in a fluctuating EFG at the iron sites with a low average value and, consequently, a small quadrupole interaction. As the temperature is lowered, the thermal motion of the vacancies decreases until the EFG, during the lifetime of the $14.4 \mathrm{keV}$ state, is nearly that of a fixed vacancy, resulting in a larger quadrupole interaction. The existence of such vacancies also explains the presence of two magnetic hyperfine fields in the Mössbauer spectra, corresponding to iron ions with and without nearestneighbour vacancies.

Based on the existence of vacancies, the following cationic composition for jacobsite is then proposed:

$$
\left(\mathrm{Mn}^{2+}\right)\left[\mathrm{Fe}_{2-x}^{3+} \mathrm{Mn}_{y}^{3+} \cdot{ }_{x-y}\right] \mathrm{O}_{4-z}^{2-} .
$$

In this distribution, $z$ is the number of oxygen vacancies per unit chemical formula, $x-y$ is the number of cation vacancies, which are presented by the full circle. The existence of cation vacancies is a consequence of charge neutrality. The charge balance in the proposed distribution requires

$$
3(x-y)=2 z .
$$

We have assumed that the hyperfine magnetic fields at the $\mathrm{Fe}^{3+}$ nuclei in the octahedral sites are a function of the occupation of the six nearest-neighbour sites by $\mathrm{O}^{2-}$. Thus, the relative intensities of the two octahedral site peaks can be estimated since they are proportional to the probabilities of the different surroundings. These probabilities are given by

$$
I(n)=\left(\begin{array}{l}
n \\
6
\end{array}\right)(1-C)^{6-n} C^{n},
$$


where $I(n)$ is the relative intensity of the octahedral site peak with $n \mathrm{O}^{2-}$ anions in the nearest-neighbour sites and $C$ is the probability that $\mathrm{O}^{2-}$ anions occupy these sites. As the jacobsite spectra show the presence of just two magnetic hyperfine fields, it is reasonable to assume that there are two possibilities: (a) octahedral sites with no nearest-neighbour vacancy, and (b) octahedral sites with one nearest-neighbour vacancy. According to our experimental results, the others are extremely small probabilities. Thus, we have $I(6) / I(5)=C / 6(1-C)$. Averaging the experimental ratio $I(6) / I(5)$ over the range of temperature used, we obtain the value of 2.9 , which yields $C=0.94$. Since the probability that $\mathrm{O}^{-2}$ occupy octahedral sites is given, based on composition (1), by $C=(4-z) / 4$, one obtains $z=0.24$ and thus eq. (2) becomes

$$
y=x-0.16 .
$$

The cationic composition can now be written as

$$
\left(\mathrm{Mn}^{2+}\right)\left[\mathrm{Fe}_{2-x}^{3+} \mathrm{Mn}_{x-0.16}^{3+} 0.16\right] \mathrm{O}_{3.76}^{2-}
$$

To evaluate $x$, let us consider the jacobsite molecular mass which is given by $M=218.36-x$. Recalling that the X-ray diffraction analysis indicated the presence of only minor amounts of $\mathrm{MnO}_{2}$ and $\mathrm{Mn}_{2} \mathrm{SiO}_{4}$, we can assume that all iron in the sample is contained in jacobsite. We also recall that X-ray fluorescence indicated $27.6 \%$ or iron in our sample. The magnetization measurements used a mass of $71.3 \mathrm{mg}$, yielding $\mu_{\text {exp }}=3 \mu_{\mathrm{B}}$ per formula unit. Since the material used was not pure jacobsite, the experimental value of $\mu$ has to be corrected. The corrected value $\mu_{\text {corr }}$ is given by $\mu_{\text {corr }}=\left(71.3 / \Delta m_{\mathrm{j}}\right) \mu_{\text {exp }}$, where $\Delta m_{\mathrm{j}}$ is the mass of jacobsite, given by $\Delta m_{\mathrm{j}}=(M / 56(2-x)) \Delta m_{\mathrm{Fe}}$, where $\Delta m_{\mathrm{Fe}}$ is the amount of iron in the sample. Then, the corrected magnetic moment per formula unit, expressed as a function of $x$, is

$$
\mu_{\mathrm{corr}}=\left[\frac{56(2-x) 213.9}{(218.36-x) 19.7}\right] \mu_{\mathrm{B}}
$$

On the other hand, taking the magnetic moments for $\mathrm{Fe}^{3+}$ and $\mathrm{Mn}^{2+}$ to be $5 \mu_{\mathrm{B}}$, for $\mathrm{Mn}^{3+}$ equal to $4 \mu_{\mathrm{B}}$, and the magnetic moments at the two distinct crystallographic sites antiparallel, we obtain, based on composition (4), another expression for the net magnetic moment of jacobsite,

$$
\mu=(4.36-x) \mu_{\mathrm{B}} .
$$

Using eqs. (5) and (6), we find $x=0.68$ and consequently from eq. (3), $y=0.52$. Therefore, the cation distribution for the jacobsite under study is given by $\left(\mathrm{Mn}^{2+}\right)\left[\mathrm{Fe}_{1.32}^{3+} \mathrm{Mn}_{0.52}^{3+} \bullet 0.16\right] \mathrm{O}_{3.76}^{2-}$, with a net magnetic moment $\mu=3.68 \mu_{\mathrm{B}}$ per formula unit. 
The magnetic hyperfine fields at $\mathrm{Fe}^{3+}$ in the octahedral sites in jacobsite, 419 and $400 \mathrm{kOe}$, are significantly smaller than the magnetic hyperfine field at $\mathrm{Fe}^{3+}$ in the octahedral sites for synthetic manganese ferrite ( $447 \mathrm{kOe})$ [4]. Bearing in mind that we are dealing with a manganese-rich ferrite, the excess manganese must go into the octahedral sublattice as $\mathrm{Mn}^{3+}$ replacing $\mathrm{Fe}^{3+}$. Since $\mathrm{Mn}^{3+}$ has a smaller magnetic moment than $\mathrm{Fe}^{3+}$, the average magnetization of the octahedral site decreases. Consequently, the magnetic hyperfine field at the iron nuclei decreases.

Finally, recent investigations by Mössbauer spectroscopy of the thermal transformations of jacobsite [14] have yielded results in agreement with the above model. It has been observed that calcination of jacobsite at a temperature of $400^{\circ} \mathrm{C}$ induces changes that result in a Mössbauer spectrum that is similar to that of synthetic manganese ferrite (fig. 2). As expected, these changes may be attributed to destruction of cation and oxygen vacancies, plus migration of ferric ions from the octahedral to the tetrahedral sublattices.

\section{Acknowledgement}

This work was supported by the Brazilian agencies CNPq and Finep, and by the US National Science Foundation.

\section{References}

[1] J.M. Hastings and L.M. Corliss, Phys. Rev. 104(1956)328.

[2] I.I. Yamzin, N.V. Belov and Y.Z. Nozik, J. Phys. Soc. Japan 17(1962)55.

[3] A.H. Eschenfelder, J. Appl. Phys. 29(1958)378.

[4] G.A. Sawatzky, F. van der Woude and A.H. Morrish, Phys. Lett. A25(1967)147.

[5] A. Maun and S. Somiya, Am. J. Sci. 260(1962)230.

[6] B. von Punge-Witteler, Z. Phys. Chem. 142(1984)239.

[7] K. Kleinstück, E. Wieser, P. Kleinert and R. Perthel, Phys. Stat. Sol. 8(1965)721.

[8] A. Murasik and G. Rouli, J. de Phys. 25(1964)522.

[9] S.E. Harrson, C.J. Kriesman and S.R. Pollack, Phys. Rev. 110(1958)844.

[10] M. Tanaka, T. Mizaguchi and Y. Aiyama, J. Phys. Soc. Japan 18(1963)1091.

[11] F.K. Lotgering and A.M. van Diepen, J. Phys. Chem. Solids 34(1973)1369.

[12] B.D. Josephson, Phys. Rev, Lett. 4(1960)341.

[13] P.A. Montano, H. Scheter, E. Cohen and J. Makovski, Phys. Rev. B9(1974)1066.

[14] M.A.B. de Araújo, T.V.V. Costa, M.C.A. Lima, H.A. Maia and V.W. Vieira, Phys. Scripta 40(1989)682. 